\title{
Knowledge, Attitudes and Self-care Practices associated with Glaucoma among Hospital Personnel in a Tertiary Care Center in North India
}

\author{
Parul Ichhpujani, Shibal Bhartiya, Manisha Kataria, Prateek Topiwala
}

\section{ABSTRACT}

Purpose: To determine the level of correct knowledge about glaucoma and attitudes toward blindness prevention and treatment and how these factors influence self-care practices among hospital personnel.

Methods: In this tertiary hospital based, cross-sectional study, a random sample of 119 staff members including 23 physicians (nonophthalmologists) and 96 nursing staff were administered a self-designed knowledge, attitudes, practice (KAP) questionnaire about glaucoma.

Results: All 119 personnel [34 (28.57\%) males; 85 (71.42\%) females] were aware of glaucoma. Most physicians $(80.76 \%)$ and nurses $(65.26 \%)$ unders tood that glaucoma was associated with a high intraocular pressure and had an effect on the optic nerve. Twenty-four percent of physicians and nurses did not know that it is important for family members of glaucoma patients to be more concerned about getting the disease. As regards 'treatment priority' between cataract, glaucoma and diabetic retinopathy; $76.91 \%$ physicians and $60 \%$ nurses placed glaucoma first. Out of total blindness, stroke or paralysis, cancer, schizo phrenia and heart disease, blindness prevention was first priority for $9(34.60 \%)$ physicians and $15(15.78 \%)$ nurses. A recent visit to an eye practitioner $(p=0.012)$ was a significant predictor of knowledge of glaucoma as a blinding disease.

Conclusion: Educating hospital workers on the symptoms of glaucoma and visual impairment can be an important step toward preventive ophthalmic care.

Keywords: Attitude, Awareness, Glaucoma, Intraocular pressure, Knowledge.

How to cite this article: Ichhpujani P, Bhartiya S, Kataria M, Topiwala P. Knowledge, Attitudes and Self-care Practices associated with Glaucoma among Hospital Personnel in a Tertiary Care Center in North India. J Current Glau Prac 2012;6(3):108-112.

\section{Source of support $\mathrm{Nil}$}

Conflict of interest: None declared

\section{INTRODUCTION}

As the world population continues to age, the public health impact of vision loss due to eye diseases will continue to grow and glaucoma would remain as the second or the third most common cause of irreversible blindness in the world. ${ }^{1}$ It has been estimated that almost $90 \%$ of glaucoma-related blindness can be prevented with early and proper treatment. ${ }^{1,2}$
It has been seen from various population-based studies that the awareness and knowledge of glaucoma among both rural and urban population is remarkably low, specially in developing countries, which has a negative impact on health seeking behavior. ${ }^{3-5}$ Given that the outreach of the health care system in developing countries remains far from optimal, it is essential that each of the health care providers be educated about glaucoma so as to reach a large sector of the population, which does not have access to a comprehensive eye care center.

Despite its significance, little information is available on knowledge, attitudes and self-care practices associated with glaucoma among hospital workers, with the solitary study in this regard being from Nigeria. ${ }^{6}$

This cross-sectional, questionnaire-based study was carried out to determine the level of knowledge about glaucoma and attitudes toward blindness prevention and treatment, and how these factors influence self-care practices among hospital personnel.

\section{METHODS}

The study was carried out at Government M edical College and Hospital, Chandigarh, a tertiary care hospital, associated with multidisciplinary undergraduate and postgraduate training programs located in an urban area of N orth India.

A self-designed nine-point questionnaire (Table 1 ) about glaucoma was administered to a randomly selected group of hospital workers. The same interviewers (PI) assessed each of the respondents in terms of their knowledge, attitudes and self-care practices associated with glaucoma.

A wareness was defined as 'having heard of glaucoma'. Knowledge was defined as when the subject had some understanding of glaucoma in terms of cause and/or symptoms; high pressure in the eye, damage to the nerve of the eye due to high pressure, damage to retina and the presence of colored haloes and pain in the eye.

Each of the subjects was first questioned whether he or she had heard of the disease entity called glaucoma. Further questions pertaining to knowledge of glaucoma were asked only if the subjects responded in the affirmative and were then asked to explain what they knew of the disease. 
Knowledge, Attitudes and Self-care Practices associated with Glaucoma among Hospital Personnel in a Tertiary Care Center

Table 1: KAP questionnaire for glaucoma

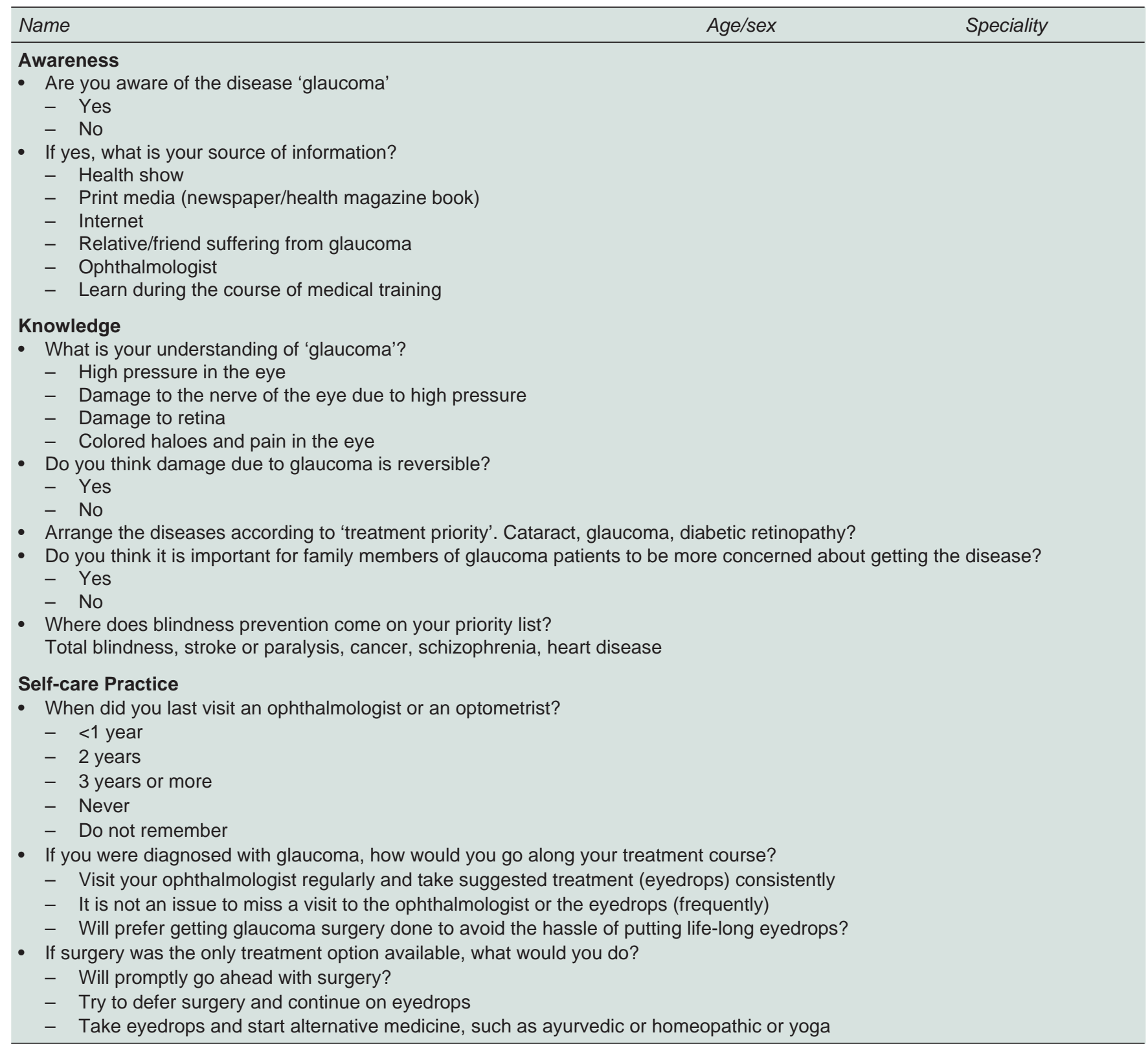

The questionnaire contained a list of possible responses in terms of high pressure in the eye, damage to the nerve of the eye due to high pressure, damage to retina and symptoms of colored haloes and pain in the eye.

The interviewer marked the response provided by the subject against the response it most closely approached on the questionnaire. In case the response did not correlate with any of the responses listed on the questionnaire, it was documented in greater detail.

Self-care practices were ascertained by a set of three questions on their attitudes toward the prevention and treatment of blindness of glaucoma.

All participants were asked to provide an estimate of their last visit to an eye practitioner that is, either an ophthal mologist or optometrist, and the duration since last visit was documented as $<1,2$ or 3 years or more years, or never.

\section{Data Analysis}

During statistical analysis, the participants were categorized as either nurses or doctors. Data was analyzed using the SPSS version 16.00 (Chicago, Illinois, USA).

The relationship between awareness of glaucoma and demographic factors, such as age, gender and education status was assessed using the Chi-square test. A two-tailed p-value of less than 0.05 was considered statistically significant. 


\section{RESULTS}

Of the 119 hospital personnel (96 staff nurses; 23 nonophthalmologist physicians) who consented to participate in the study, and answered the questionnaire, $85(71.42 \%)$ were females and 34 (28.57\%) males. Overall, 96 (80.7\%) subjects had secondary level of education while $23(19.3 \%)$ subjects had tertiary level of education.

\section{Awareness}

All 119 subjects were aware of glaucoma. The sources of information about glaucoma included health shows in $18(15.1 \%)$, print media in $21(17.6 \%)$ and the internet in $17(14.3 \%)$ subjects. A relative/friend suffering from glaucoma was the source of knowledge for 15 (12.6\%), while discussions with attending ophthalmologist and learning during the course of study (nursing/medical) accounted for $22(18.5 \%)$ and 94 (78.9\%) subjects, respectively. As is evident from the distribution, several subjects reported more than one source of information about the disease process.

\section{Knowledge}

M ost physicians ( $80.76 \%$ ) and nurses (65.26\%) understood that glaucoma was associated with a high pressure and had an effect on the optic nerve (Table 2).

Twenty-four percent of physicians and nurses did not know that it is important for family members of glaucoma patients to be more concerned about getting the disease. Forty-seven $(47,39.5 \%)$ subjects thought that the damage due to glaucoma is reversible.

A s regards 'treatment priority' between cataract, glaucoma and diabetic retinopathy; $76.91 \%$ physicians and
$60 \%$ nurses placed glaucoma first. Out of total blindness, stroke or paralysis, cancer, schizophrenia and heart disease, blindness prevention was first priority for 26 subjects (21.8\%) [9 (34.6\%) physicians; 15 (15.78\%) nurses].

The only significant predictor of knowledge of glaucoma as a blinding disease were recent visit to an ophthal mologist $(p=0.001)$; while medical profession $(p=0.227)$ and female sex $(p=0.482)$ were not found to be significantly predictive of knowledge of the disease.

\section{Self-care Practices}

Only $50(42 \%)$ subjects had visited an ophthalmologist within the past year. Overall, $92(77.3 \%)$ subjects answered that if they were diagnosed with glaucoma, they would visit their ophthal mologist regularly and take suggested treatment (eyedrops) consistently. If surgery was the only treatment option available then $103(86.6 \%)$ subjects were willing to promptly go ahead with the surgery (Table 3 ).

\section{DISCUSSION}

Promoting awareness of common eye diseases and implementing health care programs in a given community can bring forth people to have an eye examination. ${ }^{3,7}$ In a disease like glaucoma, early diagnosis and institution of treatment can result in reduction of visual impairment and blindness, since the major predictor of eventual blindness is late presentation.

$V$ arious studies have emphasized a poor awareness of eye diseases among the general population, ${ }^{4,5,7,8}$ with approximately $50 \%$ of patients with glaucoma being unaware of their condition at the time of diagnosis ${ }^{9}$ and present in the advanced stage of the disease. ${ }^{10,11}$

Table 2: Understanding of glaucoma

\begin{tabular}{ll}
\hline Understanding of glaucoma & $(\mathrm{n}=119)$ \\
\hline High pressure in the eye & $80(67.2 \%)$ \\
Damage to nerve due to high pressure & $56(47.1 \%)$ \\
Damage to retina & $26(21.8 \%)$ \\
Colored halos and pain in the eye & $24(20.2 \%)$ \\
(Some subjects gave more than 1 response) & \\
\hline
\end{tabular}

Table 3: Self-care practice along treatment course of glaucoma

\begin{tabular}{lc}
\hline Practice along treatment course of glaucoma & $(\mathrm{n}=119)$ \\
\hline Visit your ophthalmologist regularly and take suggested treatment (eyedrops) consistently & $92(77.3 \%)$ \\
Not an issue to miss a visit to the ophthalmologist or frequently miss a dose of medication & $8(6.7 \%)$ \\
Will prefer getting glaucoma surgery done to avoid the hassle of putting lifelong eyedrops? & $18(15.1 \%)$ \\
If surgery was the only treatment option available? & $103(86.6 \%)$ \\
Will promptly go ahead with surgery? & $10(8.4 \%)$ \\
Try to defer surgery and continue on eyedrops & $4(3.4 \%)$ \\
Eyedrops; start adjunctive alternative medicine & \\
\hline
\end{tabular}


It also must be kept in context that there have been few studies demonstrating the association of late presentation of glaucoma with social factors from the U nited K ingdom ${ }^{12-14}$ and India ${ }^{3}$ whereglaucomaisasignificantcause of blindness. It was observed in Moorefield's Eye Hospital Study that glaucoma diagnosis was missed by optometrists and sometimes, ophthalmologists, perhaps because a comprehensive eye examination was not performed. ${ }^{12,13}$ In fact, analysis of late attenders in the clinic revealed that people referred by any sourceother than an optometristw ho has made the correct diagnosis of glaucoma were 4.5 times morelikely to be late attenders than patients, so referred but similar in other mentioned factors. Similar findings were observed in the $B$ arbados eyestudies where visits to theoptometrists still left many patients unaware about their glaucomatous condition. ${ }^{15}$ It is therefore imperative that hospital staff be aware of glaucoma and its implications in order to improve case finding and initiation of treatment at an early stage.

In this study, a startling fact that emerged was that as many as $20 \%$ doctors and $35 \%$ nurses working in a tertairy care center affiliated to a university teaching institution did not understand that glaucoma was associated with a high pressure and had an effect on the optic nerve. A Imost 1 in 4 doctors and nurses did not realize that it is important to screen family members of glaucoma patients for the disease, since they have a higher-risk of disease. Forty percent of those interviewed actually believed that the damage due to glaucoma is reversible.

The implications of these findings are that as many as one in four nonophthalmologists and nurses may not be considered as potential sources of knowledge for patients who reach a tertiary care hospital, and in fact, if questioned, may mislead these patients.

In a similar study among doctors, nurses and nonmedical hospital staff from the Obafemi A wolowo University Teaching Hospital in Nigeria ${ }^{6}$ revealed equally disheartening results.

Almost one in two (48.8\%) subjects consisting of 38 doctors, 53 nurses and 9 nonmedical staff did not know whether visual loss due to glaucoma was permanent or reversible. Unlike our study, female gender ( $p=0.003)$, secondary or tertiary level of education $(p=0.001)$, were found to be significant predictors of knowledge of glaucoma as a blinding disease. Like in our study, a recent visit to an eye practitioner ( $p=0.012$ in N igeria vs 0.001 in our study) was a significant predictor of knowledge of glaucoma.

A Imost half the subjects in Nigeria believed that treatment of glaucoma should be given highest priority compared with other diseases, as against $61 \%$ in our study (73/119; $76.9 \%$ physicians and $60 \%$ nurses).
It must also be kept in mind that more than $20 \%$ of the subjects ( 2 doctors and 22 nurses) did not cite their medical education as the source of information about glaucoma and its implications. It must therefore be considered as a serious lacuna in the design of the education curriculum for, both, doctors and nurses.

Despite working in a hospital, with easy and free access to health care, only $42 \%$ of the subjects had visited an ophthalmologist within the past year. B arriers to seeking health care, therefore, are more than just access to affordable health care and knowledge about the disease and its implications.

Reluctance to regular visits to the ophthalmologist and the use of medication (eyedrops) was reported by more than $20 \%$ of subjects in case of a probable diagnosis with glaucoma.

In contrast, less than $15 \%$ reported a hesitation, if surgery was offered to them as the only treatment option available.

\section{CONCLUSION}

Given that the doctors and nurses in departments other than ophthalmology are often the first point of contact when patients seek medical advice, these health care providers must be well-informed or the risk of misinformation and wrong counseling is very high, even in a tertiary care center. It must be kept in mind that it is incorrect to assume adequate knowledge and appropriate attitude among nonophthalmologists and nurses, as regards glaucoma.

It is, therefore, important to emphasize on intensive eye health education and information dissemination, especially among health care professionals. Educating hospital personnel about the presentation and outcome of this ' silent thief of sight' can be an important step toward preventive ophthalmic care. Continuing medical education about glaucoma symptoms and subsequent visual impairment, therefore, must be a priority when designing programs for community outreach.

\section{REFERENCES}

1. Quigley HA, Broman A T. The number of people with glaucoma worldwide in 2010 and 2020. Br J Ophthalmol 2006;90: 262-67.

2. Quigley HA. N umber of people with glaucoma w orldwide. $\mathrm{Br}$ J Ophthalmol 1996;80:389-93.

3. Gogate P, D eshpande R, Chelerkar V, Deshpande S, D eshpande $M$. Is glaucoma blindness a disease of deprivation and ignorance? A case-control study for late presentation of glaucoma in India. Indian J Ophthalmol 2011;59:29-35.

4. Sathyamangalam RV, Paul PG, George R, Baskaran M, Hemamalini A, M adan RV, et al. Determinants of glaucoma awareness and knowledge in urban Chennai. Indian J Ophthalmol 2009;57:355-60. 
5. Krishnaiah S, Kovai V, Srinivas M, Shamanna BR, Rao GN, Thomas $R$. A wareness of glaucoma in the rural population of Southern India. Indian J Ophthalmol 2005;53:205-08.

6. A degbehingbe BO, B isiriyu $L A$. K nowledge, attitudes and selfcare practices associated with glaucoma among hospital workers in Ile-Ife, Osun State, Nigeria. Tanzan J Health Res 2008;10: 240-45.

7. Thapa SS, B erg RV , K hanal S, Paudyal I, Pandey P, M aharjan $\mathrm{N}$, et al. Prevalence of visual impairment, cataract surgery and awareness of cataract and glaucoma in B haktapur district of Nepal: The Bhaktapur Glaucoma Study. BM C Ophthalmol 2011;11:2.

8. Dandona R, Dandona L, J ohn RK, McCarty CA, Rao GN. $A$ wareness of eye diseases in an urban population in Southern India. Bull W orld Health Organ 2001;79:96-102.

9. Tielsch J M, Sommer A, K atz J, R oyall RM, Quigley HA, Javitt $J$. Racial variations in the prevalence of primary open angle glaucoma: The Baltimore Eye Survey. J Am Med Assoc 1991;369-74.

10. Dandona L, Dandona R, Srinivas M, M andal P, John RK, $M C C$ arty $C A$, et al. O pen-angle glaucoma in an urban population in Southern India: The A ndhra Pradesh eye disease study. Ophthalmology 2000;107:1702-09.

11. Dandona L, Dandona R, M andal P, Srinivas $M$, John RK, McCarty CA, Rao GN. A ngle-closure glaucoma in an urban population in Southern India. The A ndhra Pradesh eye disease study. O phthalmology 2000;107:1710-16.

12. Fraser $S, B$ unce $C$, W ormald $R$. R isk factors for late presentation in chronic glaucoma. Invest Ophthalm V is Sci 1999;40: 2251-57.

13. Fraser $S, B$ unce $C$, W ormald $R$, B runner $E$. Deprivation and late presentation of glaucoma: Case control study. BM J 2001;322:639-43.
14. Ng W S, A garwal PK, Sidiki S, M ackay L, Townend J, A zuara $B$ lanco $A$. The effect of socioeconomic deprivation on severity of glaucoma at presentation. BrJ Ophthalmol 2010;94:85-87.

15. Hennis A, Wu S, Nemesure B, Honkanen R, Leske M C. Barbados Eye Studies Group. A wareness of incident open-angle glaucoma in a population study: The Barbados Eye Studies. Ophthalmology 2007;114:1816-21.

\section{ABOUT THE AUTHORS}

\section{Parul Ichhpujani (Corresponding Author)}

A ssistant Professor, Glaucoma Facility, Department of O phthalmology Government M edical College and Hospital, Sector 32, Chandigarh India, Phone: 91-172-2601023 (Ext. 2413), Fax: 91-172-2608488 e-mail: parul77@ rediffmail.com

\section{Shibal B hartiya}

Consultant, Department of O phthalmology, Glaucoma Facility, F ortis M emorial Research Institute, Gurgaon, Haryana, India

\section{Manisha Kataria}

Glaucoma Facility, Department of Ophthalmology, Government M edical College and Hospital, Chandigarh, India

\section{Prateek Topiwala}

Glaucoma Facility, Department of Ophthalmology, Government M edical College and Hospital, Chandigarh, India 